\title{
A ESPERANÇA NA SAÚDE MENTAL: UMA REVISÃO INTEGRATIVA DA
} LITERATURA

\author{
| Ana Querido'; Maria dos Anjos Dixe ${ }^{2} \mid$
}

\section{RESUMO}

CONTEXTO: A esperança é um determinante importante da saúde mental, reconhecida por prevenir depressão e dos comportamentos suicidários e ajudar no processo de recuperação da doença mental. O conhecimento sobre a esperança na saúde mental está suportado em revisões da literatura até 2006, desconhece-se o estado da arte em relação à esperança desde então.

OBJETIVO(S): Conhecer o papel da esperança na SM das pessoas e os determinantes associados, através da análise da literatura publicada entre 2006 e 2014.

METODOLOGIA:Revisão integrativa da literatura usando como descritores esperança e saúde mental em diferentes bases de dados eletrónicas nacionais e internacionais. Foram incluídos artigos em inglês, português e espanhol, publicados entre 2006-2014, segundo critérios de seleção previamente definidos.

RESULTADOS: Após aplicação dos critérios de seleção, inclusão e exclusão dos artigos foram encontradas 26 publicações, na sua maioria anglosaxónicas com populações heterogéneas, utilizando vários métodos de investigação.

A esperança tem uma influência positiva na saúde mental das pessoas, no aumento do conforto e da qualidade de vida e na redução da depressão e suicídio, da predisposição para a dependência, e da exaustão familiar.Identificaram-se relações entre os determinantes de saúde mental e a esperança, fatores influenciadores e os contextos para a utilização da esperança.

CONCLUSÃO: Os estudos reforçam o efeito positivo da esperança na saúde mental das pessoas. A literatura fornece evidência que identifica fatores potenciadores e suporta o desenvolvimento de estratégias promotoras de esperança em saúde mental, apontando para o uso intencional da esperança em diversos contextos.

PALAVRAS-CHAVE: Esperança; Saúde mental; Doença mental; Revisão da literatura

\section{RESUMEN}

"La esperanza en la salud mental: Una revisión integrativa de la literatura"

CONTEXTO: La esperanza es un determinante importante de la salud mental, reconocida por la prevención de la depresión y el comportamiento suicida y por ayudar en el proceso de recuperación de la enfermedad mental. El conocimiento de la esperanza en la salud mental se apoya en la revisión de la literatura hasta el año 2006, se desconociendo el estado del arte actual en relación a la esperanza.

OBJETIVO(S): Conocer el papel de la esperanza en la salud mental de las personas y los determinantes asociados, a través de revisión de la literatura publicada entre 2006 y 2014.

METODOLOGÍA: Revisión integrativa utilizando descriptores tales como la esperanza y la salud mental en las diferentes bases de datos electrónicas nacionales e internacionales. Ellos incluyeron artículos en Inglés, portugués y español, publicados entre 2006-2014, de acuerdo con los criterios de selección predefinidos.

RESULTADOS: Después de aplicar los criterios de selección, la inclusión y exclusión de los artículos se encontraron 26 publicaciones, en su mayoría anglosajones con poblaciones heterogéneas, utilizando diversos métodos de investigación. La esperanza tiene una influencia positiva en la salud mental de las personas, que aumenta la comodidad y la calidad de vida, y en la reducción de la depresión y el suicidio, de la predisposición a la adicción y agotamiento familiar. Se identificaran determinantes de la salud mental en la esperanza, factores y contextos que influyen en el uso de la esperanza.

CONCLUSIÓN: Los estudios refuerzan el efecto positivo de la esperanza en la salud mental. La literatura identifica factores potenciadores y apoya el desarrollo de estrategias que promuevan la esperanza en la salud mental, señalando el uso intencional de la esperanza en diferentes contextos.

\section{DESCRIPTORES: Esperanza; Salud mental; Enfermedad men-} tal; Revisión de literatura

\begin{abstract}
"Hope in mental health: An integrative literature review"

BACKGROUND: Hope is an important determinant of mental health, recognized for preventing depression and suicidal behaviour and for the major role in recovery. Scientific knowledge regarding hope in mental health is supported by the literature review conducted in 2006. The state of the art of hope in mental health is unknown since then.

AIM: Understand the role of hope in service users' mental health and Identify mental health determinants in hope, by conducting an integrative review of the literature published between 2006 and 2014.

METHODS: Integrative literature review using Mesh terms "hope" and "mental health" in different national and international electronic data bases. We included articles in English, Portuguese and Spanish, published 2006-2014, according to pre-defined selection criteria.

RESULTS: After applying the selection, 26 publications match the criteria, mostly Anglo-Saxon, with heterogeneous populations and using different research methods. Hope has a positive influence on people's mental health, increasing comfort and quality of life, reducing depression and suicide, diminish the predisposition for addiction and family burnout. There are correlations between hope and determinants of mental health. Hope determinants were identified as well as the contexts for the use of hope.

CONCLUSION: The research studies reinforce the positive effect of hope in mental health. Literature provides evidence that identifies hope enhancing factors and supports the development of hope promoting strategies in mental health, emphasizing the intentional use of hope in different contexts.
\end{abstract}

\section{KEYWORDS: Hope; Mental health; Mental disorders; Litera- ture review}

Submetido em $06-11-2015$

Aceite em 10-03-2016

1 PhD; RN; Professora Adjunta na Escola Superior de Saúde do Instituto Politécnico de Leiria, Departamento de Enfermagem, Unidade de Investigação em Saúde, Campus 2 , Morro do Lena - Alto do Vieiro, Apartado 4137, 2411-901 Leiria, Portugal, ana.querido@ipleiria.pt

$2 \mathrm{PhD}$; Professora Coordenadora na Escola Superior de Saúde do Instituto Politécnico de Leiria, Unidade de Investigação em Saúde, Leiria, Portugal, maria.dixe@ipleiria.pt

Citação: Dixe, M.A., \& Querido, A. (2016). A esperança na saúde mental: Uma revisão integrativa da literatura. Revista Portuguesa de Enfermagem de Saúde Mental (Spe. 3), 95-101. 


\section{INTRODUÇÃO}

A esperança é um determinante importante da saúde mental (SM), reconhecida pela sua importância no processo de recovery, na abordagem da prevenção da depressão e dos comportamentos suicidários, reconhecida por ajudar no processo de recuperação da doença mental (World Health Organization [WHO], 2010). Na perspetiva de Jones (2007), a esperança realista e relevante pode constituir um potente agente terapêutico em tempo de dificuldades para as pessoas que enfrentam uma situação de crise/doença mental e as suas famílias. O papel da esperança na SM e a sua importância para a Enfermagem de saúde mental foi alvo de revisão da literatura entre 1980 e 2005, por Kohen e Cutcliffe (2007). Importa conhecer o estado da arte relativamente à temática desde então, pelo que se decidiu atualizar a revisão.

\section{METODOLOGIA}

Para responder ao objetivo geral deste estudo optou-se pelo método de revisão integrativa da literatura (RIL) por ser um método de investigação que permite a incorporação das evidências na prática clínica, suportando a tomada de decisão na metodologia de análise da investigação relevante, com vista à melhoria da prática clínica (Mendes, Silveira e Galvão, 2008). Com a finalidade de reunir e sintetizar os resultados da investigação sobre a esperança na SM, caracterizando o estado do conhecimento e identificar lacunas que precisam de ser colmatadas, cumpriram-se as 6 etapas sequenciais da metodologia (Mendes et al., 2008): definição da questão de investigação, seleção da amostra, categorização dos estudos, avaliação, interpretação dos resultados, apresentação da síntese do conhecimento.

A partir da questão Qual é o papel da esperança na SM das pessoas?, outras questões de investigação surgiram: Q1:Quais são os efeitos da esperança na SM das pessoas? Q2: Qual é a relação entre os determinantes de SM e esperança? Q3:Quais são os fatores que influenciam a esperança? Q4:Quais são as situações em que a esperança é significativa? Na seleção da amostra optámos pela pesquisa em bases de dados eletrónicas. No motor de busca EBSCO (CINAHL Plus with Full Text, MEDLINE with Full Text, MedicLatina, Cochrane Central Register of Controlled Trials, Cochrane Database of Systematic Review); ISI e ProQuest (b-on), RCAAP e SCIELO. Utilizámos a equação Hope AND mental health, no horizonte temporal 2006-2014, tendo identificado 120 artigos.
Estabeleceu-se o protocolo PICOD, condições de acesso e língua, para a definição de critérios de seleção para a inclusão e exclusão do estudos, apresentados na tabela 1 .

Tabela 1 - Critérios de inclusão e exclusão na seleção dos artigos

\begin{tabular}{|c|c|c|c|}
\hline $\begin{array}{l}\text { Critérios de } \\
\text { Seleção }\end{array}$ & Critérios de Inclusão & Critérios de Exclusão & $\begin{array}{l}\mathrm{N}^{\circ} \text { de } \\
\text { artigos } \\
\text { Excluídos }\end{array}$ \\
\hline Participantes & $\begin{array}{l}\text { Pessoas, todas as } \\
\text { idades }\end{array}$ & --- & \\
\hline Intervenções & $\begin{array}{l}\text { Estratégias de pro- } \\
\text { moção da esperança }\end{array}$ & $\begin{array}{l}\text { Esperança de vida; me- } \\
\text { dida de prognóstico } \\
\text { Conotações não clínicas }\end{array}$ & 54 \\
\hline Comparações & $\begin{array}{l}\text { Variáveis de SM; Fa- } \\
\text { tores influenciadores }\end{array}$ & & \\
\hline Resultados & $\begin{array}{l}\text { Antecedentes; Fatores } \\
\text { preditores /facilitado- } \\
\text { res, intervenções }\end{array}$ & $\begin{array}{l}\text { Falta de referência à } \\
\text { esperança }\end{array}$ & 12 \\
\hline $\begin{array}{l}\text { Desenho do } \\
\text { estudo }\end{array}$ & $\begin{array}{l}\text { Qualitativo, quanti- } \\
\text { tativo, triangulação, } \\
\text { RSL, metasíntese, } \\
\text { metanálise }\end{array}$ & & \\
\hline $\begin{array}{l}\text { Acesso ao } \\
\text { estudo }\end{array}$ & Texto integral & $\begin{array}{l}\text { Resumo; repetições, } \\
\text { estudos em curso }\end{array}$ & 24 \\
\hline $\begin{array}{l}\text { Língua do } \\
\text { estudo }\end{array}$ & $\begin{array}{l}\text { Inglês, português, } \\
\text { espanhol }\end{array}$ & Chinês & 4 \\
\hline
\end{tabular}

Após a aplicação dos critérios de seleção, realizámos a leitura completa de todos os artigos. Os dados foram extraídos com recurso a tabelas com referência ao ano do estudo, sujeitos, metodologia, amostra, variáveis, métodos de análise, conceitos relacionados e resultados, que facilitaram a categorização das evidência e resposta às questões de investigação.

\section{RESULTADOS}

Tal como é possível observar na tabela 2 os estudos selecionados são maioritariamente de países de língua inglesa $(69,2 \%)$ sendo prevalentes os norte americanos $(34,6 \%)$, a maioria publicados nos últimos 4 anos. As populações são diversas, as amostras heterogéneas, e foram utilizadas diversas metodologias. Salienta-se que metade dos estudos (50\%) foram realizados em utentes com doença mental, com prevalência do contexto comunitário. A grande maioria teve como alvo a população adulta (92,3\%), utilizadores do serviço de saúde $(69,2 \%)$, não havendo reporte de estudos em crianças com idades inferiores a 13 anos. 
Tabela 2 - Caracterização dos estudos

\begin{tabular}{|c|c|c|c|c|c|}
\hline \multicolumn{4}{|c|}{ Caracterização dos estudos n=26 } & No & $\%$ \\
\hline Ano & \multicolumn{3}{|l|}{$\begin{array}{l}2007 \\
2009 \\
2010 \\
2011 \\
2012 \\
2013 \\
2014\end{array}$} & $\begin{array}{l}4 \\
1 \\
4 \\
1 \\
6 \\
4 \\
6\end{array}$ & $\begin{array}{l}15,38 \\
3,84 \\
15,38 \\
3,84 \\
23,07 \\
15,38 \\
23,07\end{array}$ \\
\hline País & \multicolumn{3}{|l|}{$\begin{array}{l}\text { Austrália } \\
\text { Áustria } \\
\text { Canadá } \\
\text { Coreia do Sul } \\
\text { Holanda } \\
\text { Estados Unidos América } \\
\text { Irão } \\
\text { Polónia } \\
\text { Reino Unido } \\
\text { Suécia } \\
\text { Taiwan }\end{array}$} & $\begin{array}{l}3 \\
2 \\
1 \\
1 \\
1 \\
9 \\
1 \\
1 \\
5 \\
1 \\
1\end{array}$ & $\begin{array}{l}11,53 \\
7,69 \\
3,84 \\
3,84 \\
3,84 \\
34,61 \\
3,84 \\
3,84 \\
19,23 \\
3,84 \\
3,84\end{array}$ \\
\hline \multirow[t]{4}{*}{$\begin{array}{l}\text { Sujeitos do } \\
\text { estudo / } \\
\text { Contexto }\end{array}$} & \multicolumn{2}{|l|}{$\begin{array}{l}\text { Utentes adultos com diag- } \\
\text { nóstico doença psiquiátrica } \\
\text { Utentes com doença cardíaca }\end{array}$} & $\begin{array}{l}\text { Internamento } \\
\text { Comunidade } \\
\text { Hospital Dia } \\
\text { Internamento }\end{array}$ & $\begin{array}{l}5 \\
7 \\
1 \\
2\end{array}$ & $\begin{array}{l}19,23 \\
26,92 \\
3,84 \\
7,69\end{array}$ \\
\hline & \multirow[t]{2}{*}{ Cuidadores } & $\begin{array}{l}\text { Doentes } \\
\text { oncológicos }\end{array}$ & Comunidade & 1 & 3,84 \\
\hline & & $\begin{array}{l}\text { Doentes } \\
\text { mentais }\end{array}$ & Comunidade & 2 & 7,69 \\
\hline & $\begin{array}{l}\text { Profissionais na área de saúde } \\
\text { mental } \\
\text { Estudantes adultos ( }>18 \text { anos) } \\
\text { Estudantes jovens }(>13>18 \\
\text { anos) } \\
\text { Adolescentes } \\
\text { População Geral }\end{array}$ & & Comunidade & $\begin{array}{l}1 \\
5 \\
2 \\
1 \\
1 \\
2\end{array}$ & $\begin{array}{l}3,84 \\
19,23 \\
7,69 \\
3,84 \\
7,69\end{array}$ \\
\hline \multirow[t]{3}{*}{$\begin{array}{l}\text { Metodolo- } \\
\text { gia / Tipo } \\
\text { de estudo }\end{array}$} & Qualitativo & \multicolumn{2}{|c|}{$\begin{array}{l}\text { Fenomenologia } \\
\text { Grounded Theory } \\
\text { Análise temática }\end{array}$} & $\begin{array}{l}3 \\
2 \\
2\end{array}$ & $\begin{array}{l}11,53 \\
7,69 \\
7,69\end{array}$ \\
\hline & Quantitativo & \multicolumn{2}{|c|}{$\begin{array}{l}\text { Correlacional } \\
\text { Exprimental sem grupo de } \\
\text { controlo } \\
\text { Experimental randomizado } \\
\text { controlado }\end{array}$} & $\begin{array}{l}10 \\
1 \\
2\end{array}$ & $\begin{array}{l}38,36 \\
3,84 \\
7,69\end{array}$ \\
\hline & \multicolumn{3}{|c|}{$\begin{array}{l}\text { Validação de instrumentos } \\
\text { Revisão de Literatura (RSL - } 2 \text { / Metasíntese - 1) }\end{array}$} & $\begin{array}{l}3 \\
3\end{array}$ & $\begin{array}{l}11,53 \\
11,53\end{array}$ \\
\hline
\end{tabular}

Q1 - O efeito da esperança na saúde mental das pessoas Os estudos quantitativos identificaram a esperança como um forte preditor da SM (mais forte do que a doença mental) (Vending, Kettler \& Zajac, 2011). 15 estudos apresentaram modelos explicativos com recurso à regressão. A esperança tem um efeito mediador na ansiedade e depressão pós-operatória nos doentes cardíacos, contrabalançando a influência indesejada do coping maladaptativo (Ai, Pargament, Appel \& Kronfol, 2010). Medeia as esperiências espirituais, sentido de vida e bem estar psicológico, e prediz a satisfação com a vida (Wnuk \& Marcinkowski, 2011). Este efeito preditor do bem-estar subjetivo e protetor da SM foi ainda observado em adolescentes com baixos recursos económicos, em adultos de várias raças e culturas (Khodarahimi, 2014; Loo et al., 2014) e também nas famílias (Duggleby et al., 2014), prevenindo a exaustão familiar (Hernandez, Concepción \& Yamada, 2013).
Os estudos qualitativos acrescentam a importância da esperança no recovery (Eriksen; Arman, Dadidson, Sundfor \& Karlsson, 2014; Hobbs \& Baker, 2012) e no luto (Cutcliffe, 2006), apontando a relação com os profissionais como essencial à manifestação da esperança nestes processos.

\section{Q2 - A relação entre a esperança e os determinantes de} saúde mental

Os determinantes de SM são fatores que podem constituir causas ou consequências de problemas, ou mesmo atuar como protetores da SM, categorizados em domínios (Lethinen, 2008). O quadro 1 resume as correlações entre estes e a esperança, patentes nos estudos de regressão analisados. Salientam-se relações positivas entre a esperança e os determinantes de SM positiva, prevalecendo os fatores individuais, contrapondo às relações negativas com os determinantes potenciadores de doença mental. 
Quadro 1 - Relação entre a esperança e os determinantes de SM por populações estudadas

\begin{tabular}{|c|c|c|c|c|c|c|c|c|c|c|c|c|c|c|c|c|}
\hline \multirow{2}{*}{\multicolumn{3}{|c|}{$\begin{array}{l} \\
\text { Determinantes SM Cor- } \\
\text { relações com esperança }\end{array}$}} & \multirow[b]{2}{*}{ 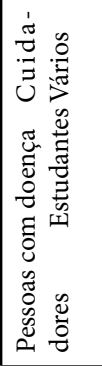 } & \multicolumn{4}{|c|}{ Pessoas com doença } & \multicolumn{4}{|c|}{ Cuidadores } & \multicolumn{3}{|c|}{ Estudantes } & \multicolumn{2}{|l|}{ Vários } \\
\hline & & & & 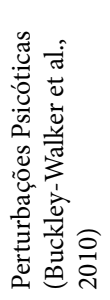 & 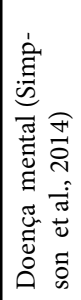 & 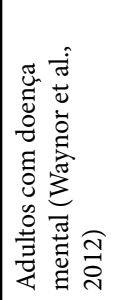 & 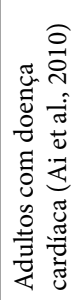 & 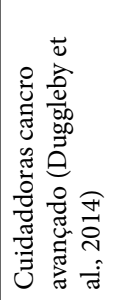 & 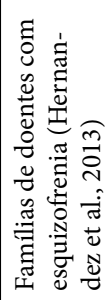 & 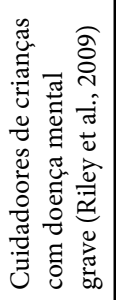 & 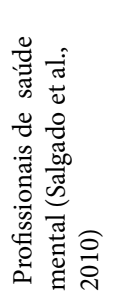 & 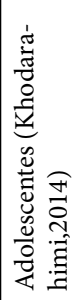 & 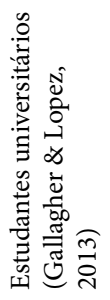 & 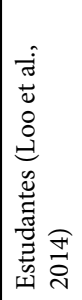 & 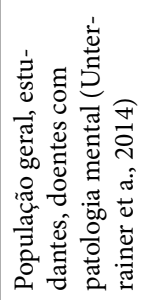 & 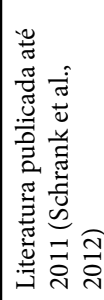 \\
\hline \multirow{26}{*}{ 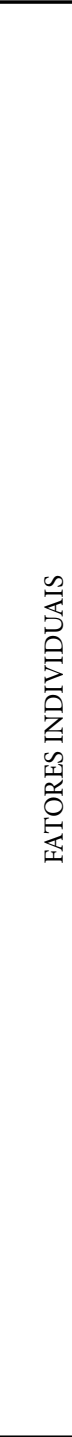 } & \multirow{9}{*}{ 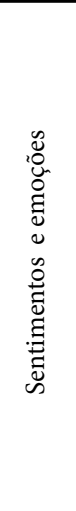 } & \multicolumn{2}{|c|}{ Otimismo } & & & & & & & & 0 & & + & & & \\
\hline & & \multicolumn{2}{|c|}{ Perda /luto } & & & & & - & & & & & & & & \\
\hline & & \multicolumn{2}{|c|}{ Sentido da vida } & & & & & & & & & & + & & + & \\
\hline & & \multicolumn{2}{|c|}{ Bem-estar } & & & & & + & & & & & + & & + & \\
\hline & & \multicolumn{2}{|c|}{ Crença - futuro } & & & & & & + & & & & & & & \\
\hline & & \multicolumn{2}{|c|}{ Gratidão } & & & & & & & & & & & + & & \\
\hline & & \multicolumn{2}{|c|}{ Perdão } & & & & & & & & & & & & + & \\
\hline & & \multicolumn{2}{|l|}{ Pena } & & & & & & & & & & & & - & \\
\hline & & \multicolumn{2}{|c|}{ Luta espiritual } & & & & - & & & & & & & & & \\
\hline & & \multicolumn{2}{|c|}{ Autoconfiança } & + & & & & + & & & & & & & & \\
\hline & $\frac{5}{2}$ & \multicolumn{2}{|c|}{ Autoeficácia } & + & & & & + & & & & & & & + & \\
\hline &. $\mathscr{\Xi}$ & \multicolumn{2}{|c|}{ Autoestima } & & & & & & & & & & & & & + \\
\hline & 区ี & \multicolumn{2}{|c|}{ Empowerment } & + & & & & & & + & & & & & & + \\
\hline & 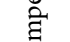 & \multicolumn{2}{|c|}{ Autonomia } & & & & & & & & & & + & & & \\
\hline & u & \multicolumn{2}{|c|}{ Resiliência } & & & & & + & + & & & & & & & \\
\hline & ت్ & Adição & & & & & & & & & & & & - & & \\
\hline & 灵 & S. de c & erência & & & & & & & & & & & & + & \\
\hline & 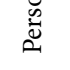 & $\begin{array}{l}\text { Copin } \\
\text { tivo }\end{array}$ & evita- & & & & - & & & & & & & & & \\
\hline & & Autoac & eitação & & & & & & & & & & + & & & \\
\hline & 㞼 & AutoP & rceção & + & & & & & & & & & + & & & \\
\hline & 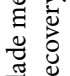 & $\begin{array}{l}\text { Autoce } \\
\text { cia }\end{array}$ & nsciên- & + & & & & & & & & & & & + & \\
\hline & 空 & Mindf & Iness & & & & & & & & & & & + & & \\
\hline & & Conhe & cimento & & & & & & & & 0 & & & & & \\
\hline & \& & Duraç & & & & & & & - & & & & & & & \\
\hline & 可 & Sintom & & & & - & - & & & & & - & & & - & - \\
\hline & $\ddot{n}-$ & Neuro & icismo & & & & & & & & & & & & - & \\
\hline & & Relaçõ & & & + & & & & & + & & & & & & \\
\hline & & Apoio & los pares & & 0 & & & & & & & & & & & \\
\hline & & $\begin{array}{l}\text { Proble } \\
\text { familia }\end{array}$ & & & & & & & & & & - & & & & - \\
\hline & & $\begin{array}{l}\text { Violên } \\
\text { familia }\end{array}$ & & & & & & & & & & - & & & & \\
\hline & & $\begin{array}{l}\text { Exaust } \\
\text { familia }\end{array}$ & & & & & & & - & - & & & & & & \\
\hline & & $\begin{array}{l}\text { Barrei } \\
\text { Empre }\end{array}$ & & & & & & & & & & & & & & - \\
\hline & & $\begin{array}{l}\text { Apoio } \\
\text { serviçc }\end{array}$ & & & & & & & + & + & & & & & & \\
\hline
\end{tabular}

Legenda: + - relações positivas; - - relações negativas; 0 - Sem evidência de relação 
Q3 - Fatores influenciadores da esperança

A relação interpessoal é o fator influenciador que se salienta da análise global dos estudos. No recovery, e em conjunto com o contexto social, determina a influência sobre a esperança das pessoas doentes, em especial as relações estabelecidas com os profissionais de SM (Cutcliffe, 2006; Hobbs \& Baker, 2012). Relações confiantes podem fortalecer a identidade, fornecendo oportunidade para o sentido e esperança, contribuindo para abertura a novas perspetivas e diminuindo a significância atribuída às manifestações de doença (Eriksen et al., 2014). A autoeficácia, autoconfiança, autoestima, resiliência, otimismo, sentido de coerência e empowerment para a gestão das situações adversas, são fatores determinantes da esperança (Gallagher \& Lopez, 2013; Schrank, Bird, Rudnick \& Slade, 2012). Já a presença de sintomas como ansiedade, depressão e ideação suicida são preditores da desesperança para os doentes (Uterrainer, Lewis \& Fink, 2014; Wanor et al., 2012) tal como a perda e o luto o são para os cuidadores (Duggleby et al., 2014). A espiritualidade influencia positivamente a esperança em diferentes contextos, com ganhos no bem estar e qualidade de vida (Schrank et al., 2012; Uterrainer et al., 2014; Loo et al., 2014). A musicoterapia e a expressão artística demonstraram efeitos positivos na estimulação da criatividade e na descoberta de forças/ recursos, contribuindo para a esperança dos doentes, através de experiências positivas de bem estar e prazer, desencadeando a motivação e a perspetiva de um futuro otimista, com ganhos na qualidade dos cuidados (Solli et al., 2013).

\section{Q4- Situações em que a esperança é significativa}

A esperança é particularmente significativa no recovery, dada a vulnerabilidade dos utentes e a suscetibilidade à presença dos profissionais de saúde, que são veículos para o fortalecimento da esperança, optimismo e empowerment através da relação interpessoal que estabelecem (Eriksen et al. 2014). Enquanto preditiva da SM, a esperança pode ser mobilizada como intervenção na prevenção da doença mental. Os estudos referem-se a intervenções pouco específicas com ganhos secundários na esperança dos doentes em contexto de recovery (Schrank et al. 2012; Simpson et al., 2014). Os achados suportam a necessidade de mais investigação relativas à utilização da esperança nas intervenções e ao suporte pelos pares e cuidadores em contexto de doença.
Não obstante, a esperança pode ser transferida dos cuidadores para as pessoas doentes, sendo significativa tanto para os cuidadores informais como para os profissionais de SM (Hobbs \& Baker, 2012; Riley et al., 2009). Em qualquer dos contextos, a investigação foi suportada por instrumentos de medida específicos, suficientemente similares e consistentes para avaliar a esperança e permitir comparações entre os resultados. O processo de validação e as propriedades psicométricas atestam a favor da sua utilização, salientando-se a Integrative Hope Scale especificamente para a população com esquizofrenia (Schrank et al., 2012).

\section{DISCUSSÃO}

A análise dos estudos selecionados permite atestar a relevância do papel da esperança na SM das pessoas. O número de estudos revela um acréscimo da investigação na área da esperança nos últimos 3 anos, comparativamente superior ao da revisão sistemática levada a cabo por Koehn e Cutcliffe (2007). As publicações revistas são dirigidas a populações com e sem doença num espectro amplo de variáveis associadas utilizando vários métodos de investigação. No entanto, na sua maioria os estudos são transversais, denotando-se a falta de evidência resultante de análises multivariadas em desenhos longitudinais. No geral foi possível identificar correlações significativas nos estudos quantitativos que corresponderam aos temas emergentes dos estudos qualitativos. Esta revisão reforça as conclusões da anterior relativamente à relevância da esperança no recovery, para os doentes, famílias e profissionais de saúde (Koehn \& Cutcliffe, 2007). Em contraponto não foi possível especificar o papel da esperança relativamente a outros grupos de diagnóstico, em parte devido à escolha das palavras-chave que não se dirigiram especificamente à patologia psiquiátrica. Este estudo analisou apenas a literatura publicada e disponível em bases de dados eletrónicas, não incluindo livros, conferências ou "literatura cinzenta",constituindo uma limitação. Ainda assim, permitiu identificar determinantes consonantes com revisões anteriores, salientando a importância dos profissionais de SM na esperança. No entanto, para estes, a esperança não determinou a melhoria das atitudes face às pessoas com doença mental grave, após um programa de formação em recovery (Salgado et al., 2010). Estes achados conduzem à necessidade de repensar as estratégias de formação dos profissionais para a capacitação para a gestão da esperança nos contextos da SM, considerando as formas como comunicam as suas esperanças sobre a recuperação dos clientes e facilitam as esperanças no recovery (Hobbs et al., 2012). 


\section{CONCLUSÃO}

A revisão integrativa da literatura realizada (2006-2014) sobre a esperança e a SM permitiu identificar efeitos da esperança na SM das pessoas, determinantes de SM relacionados com a esperança, fatores influenciadores da esperança e os contextos onde esta é significativa. Os resultados realçam a esperança como protetora da SM e preventiva da doença mental. A esperança tem efeitos positivos na SM das pessoas, prevenindo a depressão nos doentes cardíacos após a cirurgia e determinando a recuperação das pessoas com doença mental após a alta hospitalar. A evidência dos estudos transversais evidencia várias relações, mas são necessários estudos longitudinais para estabelecer causalidade. Salienta-se uma relação positiva entre a esperança e os determinantes de SM positiva, com ênfase nos fatores individuais e os atributos pessoais de esperança, a qualidade das relações, espiritualidade e bem estar. Nas pessoas com doença mental há uma correlação inversa entre a esperança e a presença de sintomas em particular ansiedade, depressão e ideação suicida. A esperança revelou-se fator de resiliência nas famílias, prevenindo a exaustão familiar nos contextos de doença mental e cancro. A análise temática dos estudos qualitativos suporta as relações evidenciadas. Salientam-se lacunas no conhecimento relativo aos modelos de esperança e às intervenções específicas desenhadas a esperança nas pessoas com problemas de SM. No entanto, foi possível identificar áreas da SM sensíveis ao efeito da esperança e estratégias promotoras de SM que podem ajudar a defenir intervenções promotoras de esperança baseadas na evidência.

\section{IMPLICAÇÕES PARA A PRÁTICA CLÍNICA}

Os resultados obtidos parecem apontar para a importância da incorporação da esperança nas estratégias colaborativas para promoção da SM e gestão da doença mental. O estudo identifica elementos e determinantes que podem ser incluídos nas futuras intervenções especialmente desenhadas para promover a esperança: enfoque na qualidade das relações estabelecidas entre os profissionais de saúde e as pessoas alvo de atenção e cuidado; facilitação das relações sociais e fortalecimento das redes de apoio; ajuda no fortalecimento dos atributos pessoais como a autoestima e autoeficácia através do empowerment; ajudar as pessoas cuidadas a assumir o controlo, a estabelecer e formular objetivos realistas; desenhar intervenções específicas de esperança, incorporando espiritualidade e bem estar.

\section{REFERÊNCIAS BIBLIOGRÁFICAS}

Ai, A., Pargament, K., Appel, H., \& Kronfol, Z. (2010). Depression following open-heart surgery:A path model involving interleukin-6, spiritual struggle, and hope under preoperative distress. Journal of Clinical Psychology, 66(10), 1057-1075. doi: 10.1002/jclp.20716

Buckley-Walker,K.,Crowe,T.\& Caputi, P.(2010).Exploring identity within the recovery process of people with serious mental illnesses. Psychiatric Rehabilitation Journal, 33(3), 219-227.

Cutcliffe, J. (2006). The principles and processes of inspiring hope in bereavement counselling: A modified grounded theory study - Part one. Journal of Psychiatric and Mental Health Nursing, 13(5), 598-603. doi: $10.1111 / j .1365-2850.2006 .01019 . x$

Koehn, C. \& Cutcliffe, J. (2007). Hope and interpersonal psychiatric/mental health nursing: A systematic review of the literature - Part one. Journal of Psychiatric and Mental Health Nursing, 14(2), 134-140. doi: $10.1111 /$ j.1365-2850.2007.01054.x

Duggleby, W., Williams, A., Holstlander, L., Thomas, R., Cooper, D., Hallstromm, L.,Gosh, S. \& O-Rourke, H. (2014). Hope of rural women caregivers of persons with advanced cancer: guilt, self-efficacy and mental health. The International Electronic Journal of Rural and Remote Health Research, Education Practise and Policy, 14(2561), 1-14.

Eriksen, K. A., Arman, M., Davidson, L., Sundfør, B., \& Karlsson, B. (2014). Challenges in relating to mental health professionals: Perspectives of persons with severe mental illness. International Journal of Mental Health Nursing, 23(2), 110-117. doi: 10.1111/inm.12024

Gallagher, M. \& Lopez, S. (2009). Positive expectancies and mental health: Identifying the unique contributions of hope and optimism. The Journal of Positive Psychology, 4(6), 548-556. doi: 10.1080/17439760903157166

Hernandez, M., Barrio, C., \& Yamada, A. M. (2013). Hope and Burden among Latino Families of Adults with Schizophrenia. Family Process, 52(4), 697-708. doi: 10.1111/famp. 12042 
Hobbs, M., \& Baker, M. (2012). Hope for recovery - how clinicians may facilitate this in their work. Journal of Mental Health, 21(2), 144-153. doi: $10.3109 / 09638237.2011 .64834$

Jones, A. (2007). Relevant hope to promote therapeutic change. Mental Health Nursing, 27(3), 14-15.

Khodarahimi, S. (2014). The Role of Family Violence on Mental Health and Hopefulness in an Iranian Adolescents Sample. Journal of Family Violence, 29(3), 259268. doi: 10.1007/s10896-014-9587-4

Lethinen, V. (2008) ed. Building up good mental health. Guidelines based on existing knowledge. Monitoring Positive Mental Health Environments Project. Helsinki, Finland: STAKES.

Loo, J. M. Y., Tsai, J.-S., Raylu, N., \& Oei, T. P. S. (2014). Gratitude, hope, mindfulness and personal-growth initiative: buffers or risk factors for problem gambling? PloS One, 9(2), e83889. doi: 10.1371/journal.pone.0083889

Mendes, K., Silveira, R.; Galvão, C. (2008). Revisão integrativa: método de pesquisa para a incorporação de evidências na saúde e na enfermagem. Texto \& Contexto - Enfermagem, 17(4), 758-764. doi: 10.1590/S010407072008000400018

Riley, S. E., Stromberg, A. J., \& Clark, J. J. (2009). Relationship between caregiver hopefulness and satisfaction with their children's mental health services. Community Mental Health Journal, 45(4), 307-315. doi: 10.1007/ s10597-009-9188-5

Salgado, J. D., Deane, F. P., Crowe, T. P., \& Oades, L. G. (2010). Hope and improvements in mental health service providers' recovery attitudes following training. Journal of Mental Health (Abingdon, England), 19(3), 243-248. doi: 10.3109/09638230903531126

Schrank, B., Bird, V., Rudnick, A., \& Slade, M. (2012). Determinants, self-management strategies and interventions for hope in people with mental disorders: systematic search and narrative review. Social Science \& Medicine, 74(4), 554-564. doi: 10.1016/j.socscimed.2011.11.008
Schrank, B., Woppmann, A., Grant Hay, A., Sibitz, I., Zehetmayer, S., \& Lauber, C. (2012b). Validation of the Integrative Hope Scale in people with psychosis. Psychiatry Research, 198(3), 395-399. doi: 10.1016/j.psychres.2011.12.052

Simpson, A., Flood, C., Rowe, J., Quigley, J., Henry, S., Hall, C., Evans, R., Scherman, P., \& Bowers, L. (2014). Results of a pilot randomised controlled trial to measure the clinical and cost effectiveness of peer support in increasing hope and quality of life in mental health patients discharged from hospital in the UK. Bio Med Central Psychiatry, 14(1), 30. doi: 10.1186/1471-244X14-30

Solli, H. P., Rolvsjord, R., \& Borg, M. (2013). Toward Understanding Music Therapy as a Recovery-Oriented Practice within Mental Health Care : A Meta-Synthesis of Service Users ' Experiences. Journal of Music Therapy, 50(4), 244-273. doi: 10.1093/jmt/50.4.244

Unterrainer, H., Lewis, A. \& Fink, A. (2014). Religious/ Spiritual Well-Being, Personality and Mental Health: A Review of Results and Conceptual Issues. Journal of Religion and Health, 53(2), 382-392. doi: 10.1007/s10943012-9642-5

Waynor, W., Gao, N., Dolce, J., Haytas, L., \& Reilly, A. (2012). The relationship between hope and symptoms. Psychiatric Rehabilitation Journal, 35(4), 345-348. doi: 10.2975/35.4.2012.345.348

Venning, A., Kettler, L., Zajac, I., Wilson, A., \& Eliott, J. (2011). Is Hope or Mental Illness a Stronger Predictor of Mental Health? International Journal of Mental Health Promotion, 13(2), 32-39. doi: $10.1080 / 14623730.2011 .9715654$

World Health Organization (WHO) (2010). mhGAP Intervention Guide for mental, neurological and substance use disorders in non-specialized health settings. Version 1.0. WHO. Acedido em http://apps.who.int/ iris/bitstream/10665/44406/1/9789241548069_eng.pdf

Wnuk, M., \& Marcinkowski, J.(2012). Do Existential Variables Mediate Between Religious-Spiritual Facets of Functionality and Psychological Wellbeing. Journal of Religion and Health, 53(1), 56-67. doi: 10.1007/s10943012-9597-6 